$\begin{array}{cc}\text { Programa de Pós-Graduação em Engenharia de Produção - PPGEP } & \\ \text { Laboratório de Qualidade de Vida - LaQVida } & \text { REVISTA BRASILEIRA DE } \\ \text { Universidade Tecnológica Federal do Paraná - UTFPR } & \text { Ponta Grossa - PR - Brasil } \\ \text { v. 04, n. 01, jan./jun. 2012, p. } 25-35 & \text { QUALIDADE DE VIDA }\end{array}$

\title{
Comparação da qualidade de vida após a cirurgia para câncer de mama associada ou não à radioterapia adjuvante
}

\section{Life quality after breast cancer surgery associated or not to the adjuvant radiotherapy}

\author{
Laíse Dantas Costa \\ Universidade Federal de Sergipe - UFS - Aracaju - Brasil \\ laisedcosta@gmail.com \\ Mariana Tirolli Rett \\ Universidade Federal de Sergipe - UFS - Aracaju - Brasil \\ mariana@ufs.br, marianatrb@gmail.com \\ Andreza Carvalho Rabelo Mendonça \\ Universidade Federal de Sergipe - UFS - Aracaju - Brasil \\ andrezinharabelo@yahoo.com.br \\ Walderi Monteiro da Silva Junior \\ Universidade Federal de Sergipe - UFS - Aracaju - Brasil \\ walderim@yahoo.com.br \\ Josimari Melo de Santana \\ Universidade Federal de Sergipe - UFS - Aracaju - Brasil \\ josimelo@infonet.com.br
}

\begin{abstract}
Resumo
O tratamento cirúrgico e adjuvante do câncer de mama muitas vezes é indispensável, mas pode trazer complicações funcionais. Da mesma forma, o emprego da radioterapia (RT) pode causar efeitos adversos e prejuízo na qualidade de vida (QV). O objetivo do estudo foi avaliar e comparar a QV de mulheres submetidas à cirurgia para o câncer de mama que realizaram ou não RT adjuvante. Foram incluídas 28 mulheres alocadas em dois grupos: grupo radioterapia (GRT) e grupo controle (GC). O GRT foi investigado antes e após a RT e o GC somente em um momento. Foi aplicado na forma de entrevista o questionário da European Organization for Research and Treatment of Cancer (EORTC), o Quality of Life Questionnaire C-30 (QLQ-C30) e o Breast Cancer Module (BR-23), específico para câncer de mama. Para a comparação entre grupos foi utilizado o teste de Mann Whitney e intragrupo o Wilcoxon Signed Rank Test, adotando significância $\mathrm{p}<0,05$. Observou-se piora da QV no item função física e desempenho funcional da escala de funções em ambos os grupos $(\mathrm{p}=0,00$ e $\mathrm{p}=0,02)$ e do item fadiga na escala de sintomas na análise final do GRT quando comparado com o $\mathrm{GC}(\mathrm{p}=0,03)$. Nos demais itens não foram encontradas diferenças significativas. Na análise dos escores do BR-23 verificou-se diferença nos sintomas no braço na comparação inicial e final do GRT com o GC ( $p=0,02$ e $p=0,02)$. Dessa forma, o emprego da RT adjuvante pode comprometer a QV destas mulheres, especialmente com relação à função física, desempenho funcional e fadiga.
\end{abstract}

Palavras-chave: câncer de mama, radioterapia, qualidade de vida, fisioterapia. 


\begin{abstract}
Surgical and adjuvant procedure for breast cancer on several occasions is indispensable, but it can cause functional complications and the use of radiotherapy (RT) can cause adverse effects and prejudice in quality of life. The objective of this study was evaluate and compare woman quality of life submitted to surgery for breast cancer and those who not received adjuvant RT. Twenty eight women were included and allocated in two different groups: radiotherapy group (RTG) and control group (CG). The GRT was investigated before and after the RT and the GC once. The questionnaire of European Organization for Research and Treatment of Cancer (EORTC), the Quality of Life Questionnaire (QLQ-C30) and the Breast Cancer Module (BR-23) were applied by an interview. Mann Whitney's test was used for comparison between groups and Wilcoxon Signed Rank Test's test was used for intragroups comparison, adopting significance $p<0.05$. There was worsening of the quality of life in item physical function and role functioning of the function scale in both groups ( $\mathrm{p}=0.00$ and $\mathrm{p}=0.02)$ and item fatigue on symptom scale in the final analysis of the GRT as compared with the GC $(\mathrm{p}=0.03)$. In all other items there were not significant differences. In analysis of the scores of the BR-23 there was difference in symptoms in the arm as in initial analysis as in the end of the GRT with GC ( $\mathrm{p}=0.02$ and $\mathrm{p}=0.02)$. So, adjuvant RT can worsened the quality of life of these women, especially in relation to physical function, role functioning and fatigue.
\end{abstract}

Keywords: breast cancer, radiotherapy, quality of life, physiotherapy.

\title{
1. Introdução
}

O câncer de mama é o segundo tipo mais prevalente de câncer, o mais comum entre as mulheres e manifesta-se como uma das principais causas de morbidade dessa população. No Brasil, de acordo com os dados do Instituto Nacional do Câncer (INCA), para o ano de 2012 foram estimados 52.680 casos novos (INCA, 2012).

A mama é um símbolo de feminilidade e sexualidade, e quando acometida pela neoplasia mamária, provoca um elevado estresse mental e consequentemente transtornos emocionais como depressão, tristeza e ansiedade. $\mathrm{O}$ tratamento envolve a cirurgia e terapias complementares como a quimioterapia, radioterapia e hormonioterapia (PICARÓ; PERLOIRO, 2005). Entretanto, as cirurgias radicais ou conservadoras ainda prevalecem. Embora sejam procedimentos terapêuticos, acarretam consequências físicas e emocionais desfavoráveis à vida da mulher, o que ocasiona um grande impacto na sua qualidade de vida (QV), especialmente no primeiro ano após a cirurgia (LEIDENIUS et al., 2005; MAKLUF; DIAS; BARRA, 2006; RIETMAN et al., 2006; DEL BIANCO et al., 2008; KOOTSTRA et al., 2008). Nesse contexto, muitas complicações físicas ocorrem simultaneamente à radioterapia (RT) para câncer de mama (HWANG et al., 2008).

A RT pode ser utilizada como terapia neoadjuvante e/ou adjuvante e tem como objetivo diminuir a recorrência locorregional (NIELSEN et al., 2006). Em algumas situações, a irradiação neoadjuvante é utilizada quando a resposta à quimioterapia primária não é satisfatória. Já em casos de recidivas ou metástase, a radioterapia é utilizada como paliativo. A irradiação também pode ser indicada em casos de dor óssea, decorrente de metástases, com o objetivo de proporcionar alívio do quadro álgico. No que se refere à ação da RT, esta é restrita à área tratada, constituindo-se em um tratamento com caráter local e regional (CAMARGO; MARX, 2000).

As reações da pele da mama durante a RT, conhecidas como radiodermite, embora reversíveis na maioria dos casos, é o efeito colateral mais comum nestas pacientes, pode afetar o programa terapêutico e até causar prejuízos na QV (PIRES; SEGRETO; SEGRETO, 2008). De acordo com Gordon et al. (2005), fisicamente, a RT desencadeia lenta reparação cicatricial, acentuada fibrose tecidual e, como consequência, um comprometimento substancial da função do membro superior. Embora seja utilizado para controle local da doença, pode contribuir para situação de cansaço, fadiga, ansiedade, tensão e nervosismo (COLLINS et al., 2004; ENGEL et al., 
2004; HWANG et al., 2008). Ainda nesse contexto, Cella e Nowinski (2002) comentam sobre os efeitos negativos da RT na QV, que pode afetar diretamente a saúde e o bem-estar.

A Organização Mundial de Saúde (OMS) define QV como sendo a percepção que o indivíduo tem de sua posição na vida, no contexto cultural, dos valores nos quais ele vive em relação aos seus objetivos, expectativas, padrões e preocupações. Qualidade de vida relacionada à saúde (QVRS) e estado subjetivo de saúde são conceitos afins, centrados na avaliação subjetiva do paciente, mais necessariamente ligados ao impacto do estado de saúde sobre a capacidade do indivíduo viver plenamente. Entretanto QV é mais geral e inclui uma variedade potencial maior de condições que podem afetar a percepção do indivíduo, seus sentimentos e comportamentos relacionados com o seu funcionamento diário, incluindo, mas não se limitando, à sua condição de saúde e às intervenções médicas (FLECK et al., 1999; OMS, 2005).

As escalas para avaliação da QV podem ser genéricas ou específicas para uma determinada condição. Instrumentos genéricos são usados para qualquer condição de saúde, sendo câncer ou não e permitem comparações entre as diversas condições, enquanto os específicos são mais sensíveis e permitem avaliar uma condição específica, por exemplo, câncer de mama Para combinar as vantagens e minimizar as desvantagens de cada tipo de instrumento, o ideal é usar, sempre que possível, a combinação de um instrumento genérico com um específico (DIAS; DIAS, 2002).

Alguns estudos como o de Collins et al. (2004) relacionaram os efeitos colaterais da RT na sobrevivência, e demonstraram que o sofrimento físico pode inibir as estratégias de enfrentamento em mulheres em tratamento radioterápico. Como muitas complicações físicas ocorrem simultaneamente à RT para câncer de mama, é importante que efetivas estratégias de prevenção sejam identificadas e bem direcionadas (COLLINS et al., 2004; HWANG et al., 2008).

Visto que o procedimento cirúrgico é indispensável para o tratamento do câncer de mama, que o emprego da RT é mandatório em alguns casos no tratamento adjuvante e que pode causar efeitos adversos durante sua aplicação, é válido reconhecer como a mulher percebe sua QV neste momento da sua vida. Incluir medidas que avaliem a QV na prática clínica facilita a comunicação entre clínicos e pesquisadores, além de ser um grande desafio interpretá-la diante do impacto físico, emocional e social causado pelo câncer de mama e seus tratamentos adjuvantes. Dessa maneira, o objetivo do presente estudo foi avaliar a QV de mulheres antes e após a realização de RT adjuvante para o tratamento do câncer de mama e compará-las com mulheres que não realizaram a RT.

\section{Materiais e Métodos}

Foi conduzido um estudo observacional do tipo transversal que avaliou mulheres tratadas cirurgicamente para câncer de mama e submetidas ou não ao tratamento adjuvante com radioterapia. Os dados foram coletados entre agosto e outubro de 2011 no Centro de Radioterapia da Fundação de Beneficência Hospital Cirurgia e no Centro de Radioterapia do Hospital de Urgência de Sergipe, ambos no município de Aracaju - SE. Os dois serviços são públicos, têm a mesma conduta terapêutica e não realizam procedimentos de retirada do linfonodo sentinela.

O estudo foi aprovado pelo Comitê de Ética em Pesquisa (CEP) da Universidade Federal de Sergipe (UFS), de acordo com o parecer 229/2011. Todas as participantes assinaram o Termo de Consentimento Livre e Esclarecido (TCLE) aceitando participar voluntariamente.

Foram incluídas mulheres submetidas à cirurgia para câncer de mama com linfadenectomia axilar e que receberam ou não RT adjuvante. Foram excluídas aquelas submetidas à RT neoadjuvante, submetidas ao tratamento há mais de 18 meses, mastectomia bilateral, colocação de prótese, realização de fisioterapia após a cirurgia, que estivessem em tratamento quimioterapêutico no período da avaliação ou que apresentassem alterações cognitivas que dificultassem a aplicação do questionário.

As participantes foram alocadas em dois grupos diferentes: mulheres submetidas ao tratamento cirúrgico e não realizaram RT adjuvante (grupo controle=GC) e mulheres submetidas ao tratamento cirúrgico e RT adjuvante (grupo radioterapia=GRT). As mesmas foram avaliadas em dois momentos: imediatamente antes de iniciar a RT e na última semana de tratamento (podendo ser 
avaliada na última ou penúltima sessão). As pacientes do GRT realizaram cinco sessões semanais de RT e a dose diária de radiação correspondia à dose total em centigray (cGy) dividida pelo número de sessões de RT. A área irradiada correspondia ao plastrão (em casos de mastectomia) ou a mama (em casos de tumorectomia ou quadrantectomia).

Inicialmente foram coletados dados pessoais e informações oncológicas (idade, estado civil, profissão, índice de massa corporal (IMC), história da doença atual e pregressa, patologias associadas, data da cirurgia, tipo de cirurgia realizada, número de sessões de radioterapia, intensidade da radiação, uso de medicamentos, dor, sinais vitais, exame físico do membro superior homolateral e contralateral à cirurgia).

Para avaliação da QV vida foi utilizado o instrumento genérico da European Organization for Research and Treatment of Cancer (EORTC), o Quality of Life Questionnaire C-30 (QLQ-C30) versão 3.0, seguido do módulo específico para câncer de mama, o Breast Cancer Module (BR-23), que foram adaptados culturalmente para o português brasileiro por Makluf (2005). Ambos os questionários possuem propriedades psicométricas adequadas, com reprodutibilidade e validade já demonstradas em diversas línguas. O QLQ-C30 é um questionário genérico composto por 30 itens, o qual incorpora cinco escalas funcionais (desempenho físico, funcional, cognitivo, emocional e social), três escalas de sintomas (fadiga, dor, náusea e vômito) e escalas de QV e estado de saúde global. Os itens simples avaliam sintomas adicionais comumente relatados por pacientes com câncer como dispnéia, perda do apetite, distúrbio do sono, constipação e diarréia, bem como o impacto financeiro da doença e tratamento. O questionário específico para câncer de mama, o BR23, engloba 23 questões que avaliam os sintomas da doença, os efeitos colaterais do tratamento, a imagem corporal, o desempenho sexual e as perspectivas futuras. São quatro possibilidades de respostas: não/pouco/moderado/muito. Há uma fórmula específica para cada escala (de função, de sintomas e de saúde global) e fórmulas iguais para calcular os escores dos itens pertencentes às mesmas escalas. Ambos os questionários apresentam escores que variam de 0 a 100 , em que 0 representa pior estado de saúde e 100 melhor estado de saúde, com exceção das escalas de sintomas que, escores maiores representam mais sintomas e pior QV.

As pacientes foram contatadas tanto por telefone quanto pessoalmente pela pesquisadora e convidadas a participarem do estudo. Nesse momento, receberam informações quanto aos objetivos do estudo e foram esclarecidas dúvidas. Após a assinatura do TCLE os questionários foram aplicados na forma de entrevista pela mesma pesquisadora em todos os momentos.

Os dados foram descritos em frequências absolutas, porcentagens, médias e desvios-padrão, medianas, mínimos e máximos. Tendo em vista que os dados não seguiram distribuição normal, as análises foram conduzidas com testes não paramétricos pelo programa BioEstat 5.0. Para comparação dos escores dos questionários entre os grupos foi utilizado o teste de Mann Whitney e para comparação intragrupo foi utilizado o teste de Wilcoxon Signed Rank Test. Foi adotado o nível de significância $\mathrm{p}<0,05$ em todas as análises.

\section{Resultados}

Foram selecionadas 30 mulheres submetidas a tratamento cirúrgico para câncer de mama associada ou não à RT adjuvante. Duas foram excluídas por motivo de afastamento do tratamento radioterapêutico, permanecendo 28 mulheres.

Quanto às características pessoais e cirúrgicas, verificou-se que os grupos eram homogêneos em relação à idade, IMC, ocupação e estado civil (Tabela 1). 
Tabela 1 - Características pessoais do grupo submetido à radioterapia (GRT) e do grupo controle (GC)

\begin{tabular}{|c|c|c|c|}
\hline & $\begin{array}{c}\text { GRT }(\mathrm{n}=13) \\
\text { Mediana (mín- } \max )\end{array}$ & $\begin{array}{c}\text { GC }(\mathrm{n}=15) \\
\text { Mediana (mín-máx) }\end{array}$ & $\mathrm{p}$ \\
\hline Idade (anos) & $55(45-73)$ & $50(31-68)$ & $0,21 *$ \\
\hline IMC $\left(\mathrm{kg} / \mathrm{m}^{2}\right)$ & $25(19,9-32)$ & $25,6(17,8-30,5)$ & $0,98 *$ \\
\hline Ocupação & $\mathrm{n}$ & $\mathrm{n}$ & \\
\hline $\begin{array}{l}\text { Atividades que exigem esforço manual } \\
\text { (do lar, doméstica, artesanato) }\end{array}$ & 10 & 13 & \\
\hline $\begin{array}{c}\text { Atividades que não exigem esforço manual } \\
\text { (professora, secretariado, serviços } \\
\text { burocráticos) }\end{array}$ & 3 & 2 & $0,96 * *$ \\
\hline \multicolumn{4}{|l|}{ Estado Civil } \\
\hline Casada & 6 & 7 & $0,98 * *$ \\
\hline Solteira & 4 & 6 & \\
\hline Viúva & 3 & 2 & \\
\hline
\end{tabular}

$\mathrm{Na}$ Tabela 2 verifica-se que a mastectomia à esquerda foi o procedimento mais frequente nos dois grupos. No GRT as sessões de radioterapia, variaram de 25 a 30 sessões e com dose correspondente a 200 (100-215) cGy por dia.

Tabela 2 - Características clínico-cirúrgicas do grupo submetido à radioterapia (GRT) e grupo controle (GC)

\begin{tabular}{|c|c|c|c|}
\hline & $\begin{array}{c}\text { GRT }(\mathrm{n}=13) \\
\mathrm{n}\end{array}$ & $\begin{array}{c}\mathrm{GC}(\mathrm{n}=15) \\
\mathrm{n}\end{array}$ & $\mathrm{p}$ \\
\hline \multicolumn{4}{|l|}{ Tipo de Cirurgia } \\
\hline Mastectomia & 6 & 13 & $0,99 * *$ \\
\hline Quadrantectomia & 5 & 2 & \\
\hline Tumorectomia & 2 & - & \\
\hline \multicolumn{4}{|l|}{ Lateralidade } \\
\hline Direito & 5 & 6 & $0,97 * *$ \\
\hline \multirow[t]{2}{*}{ Esquerdo } & 8 & 9 & \\
\hline & Mediana (mín-máx) & Mediana (mín-máx) & \\
\hline Número de linfonodos dissecados & $4(1-27)$ & $10(1-25)$ & $0,20 *$ \\
\hline Tempo da cirurgia até avaliação (semanas) & $25(12-46)$ & $8(4-46)$ & $0,00 *$ \\
\hline Número de sessões & $25(25-30)$ & - & \\
\hline Dose (cGy) & $200(100-215)$ & - & \\
\hline
\end{tabular}

Os resultados apresentados na Tabela 3 correspondem aos escores do questionário genérico do EORTC, o QLQ-C30. O escore do item desempenho funcional apresentou piora significativa quando comparado antes e após $\mathrm{RT}(\mathrm{p}=0,05)$. O mesmo foi observado no item função física tanto quando comparado antes e após a RT $(\mathrm{p}=0,00)$, quando comparado com o $\mathrm{GC}(\mathrm{p}=0,02)$. Embora não tenha apresentado diferença significativa, os itens estado de saúde global e função emocional apresentaram piora dos escores após a RT. Nos itens sobre sintomas, os escores de fadiga pioraram após a RT, mas observou-se apenas diferença significativa dos valores quando comparado o GC com aqueles após a RT ( $\mathrm{p}=0,03)$. O item náusea e dor também pioraram discretamente após a RT, não apresentando diferença estatística. Quanto à dispnéia, apenas três mulheres relataram antes e após RT e nenhuma no GC, não apresentando diferença estatística. 
Tabela 3 - Comparação dos escores do questionário QLQ-C30 antes e após a RT no grupo submetido à radioterapia (GRT) e comparação com o grupo controle (GC)

\begin{tabular}{|c|c|c|c|c|c|c|}
\hline EORTC QLQ-C30 & $\begin{array}{l}\text { Antes da RT } \\
\text { Mediana } \\
\text { (mín-máx) }\end{array}$ & $\begin{array}{l}\text { Após a RT } \\
\text { Mediana } \\
\text { (mín-máx) }\end{array}$ & $\begin{array}{c}\text { GC } \\
\text { Mediana } \\
\text { (mín-máx) }\end{array}$ & $\mathrm{p} 1$ & $\begin{array}{c}\mathrm{p} \\
\mathrm{p} 2\end{array}$ & p3 \\
\hline Estado de Saúde Global & $83,3(8,3-100)$ & $\begin{array}{l}75(33,3-100) \\
7 \text { cões }\end{array}$ & $83,3(50-100)$ & 0,21 & 0,66 & 0,21 \\
\hline $\begin{array}{c}\text { Função física } \\
\text { Desempenho funcional }\end{array}$ & $\begin{array}{l}80(66,6-100) \\
83,3(50-100)\end{array}$ & $\begin{array}{c}66,6(40-100) \\
66,6(33,3-100)\end{array}$ & $\begin{array}{c}86,6(66,6-100) \\
83,3(0-100)\end{array}$ & $\begin{array}{l}0,00 * \\
0,05 *\end{array}$ & $\begin{array}{l}0,61 \\
0,40\end{array}$ & $\begin{array}{c}0,02 * \\
0,66\end{array}$ \\
\hline $\begin{array}{l}\text { Função cognitiva } \\
\text { Função emocional } \\
\text { Função social }\end{array}$ & $\begin{array}{c}83,3(16,6-100) \\
75(0-100) \\
83,3(50-100)\end{array}$ & $\begin{array}{c}100(0-100) \\
66,6(8,3-100) \\
83,3(16,6-100)\end{array}$ & $\begin{array}{c}100(33,3-100) \\
66,6(0-100) \\
100(0-100)\end{array}$ & $\begin{array}{l}0,88 \\
0,27 \\
0,50\end{array}$ & $\begin{array}{l}0,51 \\
0,42 \\
0,69\end{array}$ & $\begin{array}{l}0,92 \\
0,89 \\
0,39\end{array}$ \\
\hline \multicolumn{7}{|c|}{ Escalas de Sintomas } \\
\hline Fadiga & $11(0-100)$ & $44,4(0-86,6)$ & $20(0-100)$ & 0,10 & 0,48 & $0,03 *$ \\
\hline $\begin{array}{l}\text { Náusea } \\
\text { Dor }\end{array}$ & $\begin{array}{c}0(0-100) \\
16,6(0-100)\end{array}$ & $\begin{array}{c}16,6(0-50) \\
33,3(0-83,3)\end{array}$ & $\begin{array}{l}0(0-100) \\
0(0-100)\end{array}$ & $\begin{array}{l}0,23 \\
0,21\end{array}$ & $\begin{array}{l}0,24 \\
0,54\end{array}$ & $\begin{array}{l}0,98 \\
0,18\end{array}$ \\
\hline Dispnéia & $0(0-66,6)$ & $0(0-100)$ & $0(0-0)$ & 0,89 & 0,30 & 0,30 \\
\hline Insônia & $33,3(0-100)$ & $33,3(0-100)$ & $33,3(0-66,6)$ & 0,89 & 0,69 & 0,42 \\
\hline Falta de apetite & $0(0-100)$ & $33,3(0-100)$ & $0(0-100)$ & 0,26 & 0,58 & 0,59 \\
\hline Constipação & $0(0-100)$ & $0(0-100)$ & $0(0-100)$ & 0,31 & 0,67 & 0,38 \\
\hline Diarréia & $0(0-100)$ & $0(0-66,6)$ & $0(0-100)$ & 0,59 & 0,71 & 0,98 \\
\hline Dificuldade financeira & $0(0-100)$ & $33,3(0-100)$ & $33,3(0-100)$ & 0,46 & 0,35 & 0,98 \\
\hline
\end{tabular}

*p< 0,05; p1 Teste de Wilcoxon Signed Rank Test; p2 e p3 Mann Withney; p1: RT inicial x RT final; p2: RT inicial x CT; p3: RT final x CT.

Fonte: Autoria própria (2011).

No questionário específico, o BR-23, verificou-se piora dos escores referentes à imagem corporal, efeitos sistêmicos da terapia e sintomas na mama quando comparado antes e após a RT, mas não foi observada diferença estatística. Quando comparado os sintomas no braço, não foi observada diferença antes e após a RT e o GC apresentou escores significativamente superiores nos dois momentos avaliados $(\mathrm{p}=0,02$ e $\mathrm{p}=0,02$, respectivamente). Após a $\mathrm{RT}$, nenhuma mulher queixou-se da função sexual e em nenhum momento nenhuma mulher referiu queda de cabelo (Tabela 4).

Tabela 4 - Comparação dos escores do questionário específico para câncer de mama BR-23 do grupo submetido à radioterapia (GRT) e do grupo controle (GC)

\begin{tabular}{|c|c|c|c|c|c|c|}
\hline EORTC BR-23 & $\begin{array}{l}\text { Antes da RT } \\
\text { Mediana } \\
\text { (mín-máx) }\end{array}$ & $\begin{array}{c}\text { Após a RT } \\
\text { Mediana } \\
\text { (mín-máx) }\end{array}$ & $\begin{array}{c}\text { GC } \\
\text { Mediana } \\
\text { (mín-máx) }\end{array}$ & $\mathrm{p} 1$ & $\begin{array}{c}\mathrm{p} \\
\mathrm{p} 2\end{array}$ & p3 \\
\hline \multicolumn{7}{|c|}{ Escalas de Função } \\
\hline Imagem corporal & $83,3(58,3-100)$ & $75(0-100)$ & $83,3(13,3-100)$ & 0,08 & 0,62 & 0,43 \\
\hline Função sexual & $0(0-66,6)$ & $0(0-100)$ & $0(0-66,6)$ & 0,46 & 0,46 & 0,94 \\
\hline Prazer sexual & $66,6(33,3-66,6)$ & Não se aplica & $66,6(33,3-66,6)$ & - & - & 1,00 \\
\hline Perspectiva futura & $33,3(0-100)$ & $33,3(0-100)$ & $66,6(0-100)$ & 0,75 & 1,00 & 0,76 \\
\hline \multicolumn{7}{|c|}{ Escalas de Sintomas } \\
\hline Efeitos sistêmicos da terapia & $13,3(0-71,4)$ & $23,8(4,7-71,4)$ & $28,5(4,7-80)$ & 0,15 & 0,34 & 0,71 \\
\hline Sintomas na mama & $8,3(0-41,6)$ & $25(8,3-75)$ & $25(0-58,3)$ & 0,08 & 0,18 & 0,87 \\
\hline Sintomas no braço & $11(0-76,6)$ & $10(0-77,7)$ & $33,3(0-100)$ & 0,95 & $0,02 *$ & $0,02 *$ \\
\hline Problemas com a queda do & - & - & - & - & - & - \\
\hline
\end{tabular}
cabelo

*p< 0,05; p1 teste de Wilcoxon Signed Rank Test; p2 e p3 Mann Withney; p1: RT inicial x RT final; p2: RT inicial x CT; p3: RT final x CT; - valor não foi considerado, pois não houve resposta.

Fonte: Autoria própria (2011). 


\section{Discussão}

Os efeitos da doença e do tratamento em mulheres com câncer de mama sobre a QV são bastante conhecidos, especialmente no primeiro ano após a cirurgia (MAKLUF, 2005; LEIDENIUS et al., 2005; RIETMAN et al., 2006; DEL BIANCO et al., 2008; KOOTSTRA et al., 2008). Entretanto, para muitas mulheres esses efeitos podem perdurar por períodos mais longos (BACK et al., 2005). E nesse contexto, a radioterapia surge como um fator ampliador afetando diretamente a saúde e o bem-estar (CELLA; NOWINSKI, 2002).

Durante a RT são observadas reações de pele como a radiodermite, além de queixas de dor e alteração da amplitude de movimento do ombro, que podem interferir negativamente na QV (GORDON et al., 2005; PIRES; SEGRETO; SEGRETO, 2008; VELLOSO; BARRA; DIAS, 2009). Além disso, pode levar a interrupção temporária do tratamento em 10\% a 30\% dos casos (BESE; HENDRY; JEREMIC; 2007; BUCHHOLZ, 2009). De acordo com Gordon et al. (2005), fisicamente, a RT desencadeia lenta reparação cicatricial, acentuada fibrose tecidual e, como consequência, comprometimento da função do membro superior. Esses achados vão de encontro aos estudos de Thomas-Maclean et al. (2008) que retrataram a RT como uma possível causadora de morbidade no braço, linfedema e também prejuízo da funcionalidade do membro superior. Os achados do presente estudo corroboram com esses autores, tendo em vista que os escores de função física pioraram após a RT, refletindo a dificuldade em realizar as atividades de vida diária como carregar sacolas, vestir-se e banhar-se, que estão presentes no questionário genérico utilizado.

É interessante observar que os escores encontrados antes da RT no presente resultado foram similares aos demonstrados por outro estudo nacional (LOTTI, 2007) e estudo internacional (REIDUNSDATTER et al., 2011), principalmente, no que se refere à função física e desempenho funcional na escala de funções do QLQ-C30. Na escala de sintomas os itens fadiga, náusea e dor foram inferiores aos escores de Lotti (2007), e similar apenas no item dor quando relacionado ao de Reidunsdatter et al. (2011).

Embora os sintomas tenham aumentado após a RT, verificou-se diferença significativa apenas com relação à fadiga e aos sintomas no braço quando comparada a avaliação após a RT com o GC. Nesse contexto, observou-se que o tempo em semanas desde a cirurgia até a avaliação das pacientes do GC foi menor quando comparadas às do GRT. Supõe-se que pelo fato de que as pacientes do GC terem sido avaliadas no pós-operatório mais recente, a presença dos sintomas no braço poderia estar relacionada aos efeitos da cirurgia. Já no GRT, os sintomas poderiam estar relacionados com o tratamento adjuvante já que estas mulheres normalmente esperam alguns meses para começar o tratamento, sugerindo que os sintomas relacionados à cirurgia já estariam minimizados. Isso corrobora com os achados de Lotti (2007), que encontrou pior escore relacionado aos sintomas no braço no pós-operatório imediato quando comparado com o pós-operatório tardio. Acrescidos disso, após a RT os escores dos sintomas no braço foram similares aos encontrados por Reidunsdatter et al. (2011) e Velloso, Barra e Dias (2009), sendo este último considerando apenas mulheres submetidas à técnica do linfonodo sentinela.

Vale ressaltar que antes da RT quatro mulheres não referiram nenhum sintoma na mama e após a RT, todas apresentaram queixas, mesmo que 'pouco' e não foi verificada diferença significativa em nenhum dos momentos avaliados. Dubernard et al. (2004) encontraram maior morbidade e consequentemente piora da $\mathrm{QV}$, principalmente das mulheres mais jovens. Makluf, Dias e Barra (2006) supõem que isso pode ser atribuído pelo fato de mulheres mais jovens terem maior dificuldade de adaptação após câncer da mama, provavelmente porque as prioridades individuais variam com a idade e os diferentes momentos da vida. Ou ainda pelo fato de que as mulheres mais velhas valorizam menos a mama e a feminilidade, e têm mais resiliência às adversidades da vida. Entretanto, de acordo com os resultados do presente estudo, no GC a função física foi melhor do que a encontrada no GRT e também foram observados piores escores relacionados aos sintomas. Acredita-se que isso possa estar relacionado ao tempo desde a cirurgia até a avaliação e não apenas à idade das pacientes. Como as mulheres avaliadas apresentaram idade semelhante, inferências ficam limitadas. 
É esperado que durante o período do tratamento adjuvante, as mulheres queixem-se de sintomas como dor, fadiga, falta de ar e insônia (GRAYDON, 1994). Entretanto, no presente estudo nenhuma mulher queixou-se de dispnéia, mas a dor e a fadiga foram relatadas e aumentaram após a RT. Cinco mulheres não relataram fadiga antes da RT e após, 11 tinham esta queixa. Adicionalmente, foram verificados escores significativamente superiores de fadiga após a RT quando comparado com o GC. A literatura aponta que a fadiga é comum em pacientes com câncer de mama e que pode exacerbar durante a RT, determinando prejuízo na QV (SERVAES et al., 2002; ISHIKAWA; DERCHAIN; THULER, 2005). Em 2009, Cadmus et al. (2009) observaram que imediatamente após o término da RT, a maioria das mulheres $(70 \%)$ vivenciou fadiga leve ou moderada e algumas (30\%) apresentaram fadiga severa a intolerável. No QLQ-C30 a fadiga é avaliada questionando-se a necessidade de repouso, a sensação de cansaço e de fraqueza. Assim, supõe-se que tais achados possam estar relacionados ao deslocamento e ao dispêndio de tempo, tendo em vista que muitas mulheres eram do interior do estado, precisavam sair de suas cidades e viajar por algumas horas até chegar ao centro de radioterapia, o que poderia levar ao cansaço e necessidade de descanso.

Há evidências de que muitas mulheres ainda continuam com fadiga depois do tratamento, e a fadiga severa está associada com fatores físicos, psicológicos, sociais, cognitivos e comportamentais que afetam a vida diária (SERVAES et al., 2002). Portanto, concorda-se com Oliveira et al. (2010) que propõem a implementação de um programa de exercícios fisioterapêuticos direcionados às pacientes submetidas à radioterapia, tendo em vista os benefícios na QV durante a RT e seis meses depois. A manutenção de escores melhores durante a RT e no período de seguimento é encorajador e sugere que a melhora não é transitória (OLIVEIRA et al., 2010).

Apesar de ter sido observado prejuízo da QV das mulheres avaliadas, as mesmas foram avaliadas num curto período de tempo, considerando apenas os efeitos imediatos da RT. Acredita-se que estudos com delineamentos mais rigorosos e com tempo de seguimento maior poderão esclarecer os efeitos tardios da RT, uma vez que estes efeitos podem não diminuir ao longo do tempo ou até mesmo algumas complicações surgirem tardiamente (DUBERNARD et al., 2004; CARLING et al., 2010; REIDUNSDATTER et al., 2011; SHARP et al., 2011). Além disso, a implementação de um programa de fisioterapia é de fundamental importância para estas mulheres e para os profissionais e serviços envolvidos com a oncologia mamária.

Verificou-se prejuízo na qualidade de vida das mulheres avaliadas e após a radioterapia adjuvante encontrou-se piores escores nos itens relacionados à função física, desempenho funcional e fadiga. Isso demonstra a importância de avaliar a QV na prática clínica, que é uma importante ferramenta para desenvolvimento de estratégias tanto para a prevenção quanto para o tratamento das morbidades causadas pelo tratamento do câncer de mama na QV.

\section{Agradecimentos}

Às equipes do Centro de Radioterapia da Fundação de Beneficência Hospital Cirurgia e do Centro de Radioterapia do Hospital de Urgência de Sergipe, em especial à fisioterapeuta Karla Mendonça por facilitar o acesso às pacientes e disponibilizar o espaço para a realização do estudo, e às bravas mulheres que aceitaram participar voluntariamente desse estudo.

\section{Referências}

BACK, M. et al. Absence of adverse early quality of life outcomes of radiation therapy in breast conservation therapy for early breast cancer. Australasian Radiology, v. 49, n. 1, p. 39-43, 2005.

BESE, N. S.; HENDRY, J.; JEREMIC, B. Effects of prolongation of overall treatment time due to unplanned interruptions during radiotherapy of different tumor sites and practical methods for 
compensation. International Journal of Radiation Oncology Biology Physics, v. 68, n. 3, p. 554$561,2007$.

BUCHHOLZ, T. A. Radiation therapy for early-stage breast cancer after breast-conserving surgery. New England Journal of Medicine, v. 360, p. 63-70, 2009.

CADMUS, L. A. et al. Exercise and quality of life during and after treatment for breast cancer: results of two randomized controlled trials. Psychooncology, v. 18, n. 4, p. 343-352, 2009.

CAMARGO, M. C.; MARX, A. G. Reabilitação física no câncer de mama. 1. ed. São Paulo: Roca, 2000.

CARLING, M. et al. Quality of life after breast radiotherapy. The Lancet Oncology, v. 11, n. 7, p. $612,2010$.

CELLA, D.; NOWINSKI, C. J. Measuring quality of life in chronic illness: the functional assessment of chronic illness therapy measurement system. Archives of Physical Medicine and Rehabilitation, v. 83, n. 12 suppl 2, p. 10-17, 2002.

COLLINS, L. G. et al. Perceptions of upper-1. body problems during recovery from breast cancer treatment. Support Care Cancer, v. 12, n. 2, p.106-113, 2004.

DEL BIANCO, P. et al. Morbidity comparison of sentinel lymph node biopsy versus conventional axillary lymph node dissection for breast cancer patients: results of the sentinella-GIVOM Italian randomized clinical trial. European Journal of Surgical Oncology, v. 34, n. 5, p. 508-513, 2008.

DIAS, R. C.; DIAS, J. M. D. Avaliação da qualidade de vida relacionada à saúde em idosos com osteoartrite de joelhos. Revista Brasileira de Fisioterapia, São Carlos, v. 6, n. 3, p. 1-7, 2002.

DUBERNARD, G. et al. Quality of life after sentinel lymph node biopsy in early breast cancer. European Journal of Surgical Oncology, v. 30, n. 7, p. 728-734, 2004.

ENGEL, J. et al. Quality of life following breast-conserving therapy or mastectomy: results of a 5year prospective study. The Breast Journal, v. 10, n. 3, p. 223-231, 2004.

FLECK, M. P. A. et al. Desenvolvimento da versão em português do instrumento de avaliação de qualidade de vida da OMS (WHOQOL-100). Revista Brasileira de Psiquiatria, v. 21, n. 1, p. 1928, 1999.

GORDON, L. G. et al. The impact of rehabilitation support services on health-related quality of life for women with breast cancer. Breast Cancer Research and Treatment, v. 93, n. 3, p. 217-226, 2005.

GRAYDON, J. E. Women with breast cancer: their quality of life following a course of radiation therapy. Journal of Advanced Nursing, v. 19, n. 4, p. 617-622, 1994.

HWANG, J. H. et al. Effects of supervised exercise therapy in patients receiving radiotherapy for breast cancer. Yonsei Medical Journal, v. 49, n. 3, p. 443-450, 2008.

INCA (Instituto Nacional De Câncer). Câncer de Mama. Rio de Janeiro, jan. 2012. Disponível em: <http://www2.inca.gov.br/wps/wcm/connect/tiposdecancer/site/home/mama>. Acesso em: 15 jan. 2012. 
ISHIKAWA, N. M.; DERCHAIN, S. F. M.; THULER, L. C. S. Fadiga em pacientes com câncer de mama em tratamento adjuvante. Revista Brasileira de Cancerologia, Rio de Janeiro, v. 51, n. 4, p. 313-318, out./nov./dez. 2005.

KOOTSTRA, J. et al. Quality of life after sentinel lymph node biopsy or axillary lymph node dissection in stage I/II breast cancer patients: a prospective longitudinal study. Annals of Surgical Oncology, v. 15, n. 9, p. 2533-2541, 2008.

LEIDENIUS, M. et al. The consequences of long-time arm morbidity in node-negative breast cancer patients with sentinel node biopsy or axillary clearance. Journal of Surgical Oncology, v. 92, n. 1, p. 23-31, 2005.

LOTTI, R. C. B. Qualidade de vida de mulheres submetidas ao tratamento de câncer da mama. 2007. 75 f. Dissertação (Mestrado em Ciências da Saúde) - Programa de Pós-Graduação em Ciências da Saúde, Instituto de Previdência dos Servidores do Estado de Minas Gerais, Belo Horizonte.

MAKLUF, A. S. D. Avaliação das propriedades psicométricas dos instrumentos Quality of life questionnaire (EORTC-QLQC30) e Breast cancer module (QLQ-BR23) para avaliação da qualidade de vida em mulheres operadas por câncer de mama. 2005. 77 f. Dissertação (Mestrado em Ciências da Reabilitação) - Programa de Pós-Graduação em Ciências da Reabilitação da Escola de Educação Física, Fisioterapia e Terapia Ocupacional, Universidade Federal de Minas Gerais, Belo Horizonte.

MAKLUF, A. S. D.; DIAS, R. C.; BARRA, A. A. Avaliação da qualidade de vida em mulheres com câncer da mama. Revista Brasileira de Cancerologia, Rio de Janeiro, v. 52, n. 1, p. 49-58, jan./fev./mar. 2006.

NIELSEN, H. M. et al. Loco-regional recurrence after mastectomy in high-risk breast cancer-risk and prognosis. An analysis of patients from the DBCG 82 b\&c randomization trials. Radiotherapy and Oncology, v. 79, n. 2, p.147-155, 2006.

OLIVEIRA, M. M. F. et al. Upper limbs exercises during radiotherapy for breast cancer and quality of life. Revista Brasileira de Ginecologia e Obstetrícia, Rio de Janeiro, v. 32, n. 3, p. 133-138, mar. 2010.

ORGANIZAÇÃO MUNDIAL DE SAÚDE. Porto Alegre: UFRGS; c1998-2004 [citado em 9 set 2005]. Divisão de saúde mental grupo WHOQOL. Disponível em: <http:/www.ufrgs.br/psiq/Whoqol>. Acesso em: 15 ago. 2011.

PICARÓ, P.; PERLOIRO, F. A evidência da intervenção precoce da fisioterapia em mulheres mastectomizadas: estudo comparativo. EssFisiOnline. [S.1.], Setubal, v. 1, n. 2, mar. 2005.

PIRES, A. M. T.; SEGRETO, R. A.; SEGRETO, H. C. RTOG criteria to evaluate acute skin reaction and its risk factors in patients with breast cancer submitted to radiotherapy. Revista Latino-Americana de Enfermagem, Ribeirão Preto, v. 16, n. 5, set./out. 2008.

REIDUNSDATTER, R. J. et al. Early effects of contemporary breast radiation on health-related quality of life - Predictors of radiotherapy-related fatigue. Acta Oncologica, v. 50, n. 8, p. 11751182, 2011. 
RIETMAN, J. S. et al. Long term treatment related upper limb morbidity and quality of life after sentinel lymph node biopsy for stage I or II breast cancer. European Journal of Surgical Oncology, v. 32, n. 2, p. 148-152, 2006.

SERVAES, P. et al. Determinants of chronic fatigue in disease-free breast cancer patients: a crosssectional study. Annals of Oncology, v. 13, n. 4, p. 589-598, 2002.

SHARP, L. et al. Frequency and severity of skin reactions in patients with breast cancer undergoing adjuvant radiotherapy, the usefulness of two assessment instruments - A pilot study. European Journal of Cancer, v. 47, n. 18, p. 2665-2672, 2011.

THOMAS-MACLEAN, R. L. et al. Arm morbidity and disability after breast cancer: new directions for care. Oncology Nursing Forum, v. 35, n. 1, p. 65-71, 2008.

VELLOSO, F. S. B.; BARRA, A. A.; DIAS, R. C. Morbidade de membros superiores e qualidade de vida após a biópsia de linfonodo sentinela para o tratamento do câncer de mama. Revista Brasileira de Cancerologia, Rio de Janeiro, v. 55, n. 1, p. 75-85, jan./fev./mar. 2009. 